\title{
A mathematical approach to virus therapy of glioblastomas
}

\author{
Victor Lopez de Rioja (i), Neus Isern and Joaquim Fort
}

\begin{abstract}
Background: It is widely believed that the treatment of glioblastomas (GBM) could benefit from oncolytic virus therapy. Clinical research has shown that Vesicular Stomatitis Virus (VSV) has strong oncolytic properties. In addition, mathematical models of virus treatment of tumors have been developed in recent years. Some experiments in vitro and in vivo have been done and shown promising results, but have been never compared quantitatively with mathematical models. We use in vitro data of this virus applied to glioblastoma.

Results: We describe three increasingly realistic mathematical models for the VSV-GBM in vitro experiment with progressive incorporation of time-delay effects. For the virus dynamics, we obtain results consistent with the in vitro experimental speed data only when applying the more complex and comprehensive model, with time-delay effects both in the reactive and diffusive terms. The tumor speed is given by the minimum of a very simple function that nonetheless yields results within the experimental measured range.

Conclusions: We have improved a previous model with new ideas and carefully incorporated concepts from experimental results. We have shown that the delay time $\tau$ is the crucial parameter in this kind of models. We have demonstrated that our new model can satisfactorily predict the front speed for the lytic action of oncolytic VSV on glioblastoma observed in vitro. We provide a basis that can be applied in the near future to realistically simulate in vivo virus treatments of several cancers.
\end{abstract}

Reviewers: This article was reviewed by Yang Kuang and Georg Luebeck. For the full reviews, please go to the Reviewers' comments section.

Keywords: Biophysics, Oncolytic virus, VSV, Glioblastoma, Front propagation speed, Reaction-diffusion equations

\section{Background}

Since early last century, viruses have been studied as experimental agents for cancer treatment. The medical interest in the field has fluctuated during this period, reaching a fever pitch in the past two decades. It was in the early 1990s, when recombinant DNA technology became standard, that virus engineering could provide scientific furtherance to virotherapy. Then, oncolytic viruses appeared to be a treatment of tremendous potential and scientists started manipulating them to target cancerous cells more specifically. This culminated in the first marketing approval of an oncolytic virus, granted by the Chinese government in November of 2005 [1]. Very recently, improvements in patient survival have led to

*Correspondence: victor.lopezd@udg.edu

ICREA/Complex Systems Laboratory, Departament de Física, Universitat de Girona, 17071 Girona, Catalonia, Spain endorsements of other oncolytic virus in Europe and the US [2]. In parallel, mathematical models of virus treatment of tumors have been developed [3-5]. However, even with this new ability to engineer viral genomes, a realistic therapeutic frontrunner has yet to emerge.

\section{Experimental background}

Among a variety of aggressive and deadly brain tumors we could highlight the glioblastoma. GBM is the most common and malignant brain cancer. Usually, treatment relies on chemotherapy, radiation and surgery. However these treatments are ineffective and the median survival time of a patient is no longer than 15 months (4 to 5 months without health care), due to multifocality of the disease, infiltrative growth and substantial tumor genotypic variability, among other factors [6, 7]. So, nowadays there are no known medical or surgical approaches that constitute 
an effective treatment of GBM, and for this reason it is widely considered that the treatment of GBM is likely to benefit from oncolytic virus therapy.

Oncolytic viruses-including retroviruses, herpesviruses and adenoviruses - are an emerging therapy tool for cancers that currently lack effective treatment [8]. The efficiency of different viruses against various tumor cell lines have been studied with in vitro and in vivo experiments $[9,10]$. Of these, vesicular stomatitis virus (VSV) has been shown in laboratory studies to have excellent capabilities to become one of the most valuable candidates for virotherapy, due to its very fast lytic cycle and its rapid oncolytic action. In addition, VSV is an enveloped, negative-strand RNA rhabdovirus that can infect a wide variety of species including mice and humans, though it is usually asymptomatic for human beings [11]. Therefore, the anticancer activity of mouse models can be transferable to human trials [12]. This fact makes VSV a strong oncolytic candidate and it has been used in preclinical studies of numerous cancer types, like glioblastomas.

Hence, we focus our attention on the development of a mathematical model of the VSV-GBM virus-tumor system. In the absence of in vivo data, all of the parameter values that we will introduce in the model are extracted from in vitro VSV-GBM experiments. Our main objective is to develop a simple model that can reproduce the VSV-GBM dynamics and explain satisfactorily the experimental in vitro propagation speeds.

\section{Previous mathematical approaches}

The most basic mathematical model of the competition between populations was constructed by Alfred J. Lotka and Vito Volterra in 1925 and 1926 independently [13]. For years their model was improved and adapted to different parasite-host systems, including virus infections [14-17]. Nevertheless, we are interested in a specific model which studies the dynamics of an oncolytic virus through a tumor cell population.

In Ref. [5], Wodarz et al. noted that the few previous reaction-diffusion models of oncolytic virus spread $[18,19]$ include, in addition to basic spatial dynamics, one or more additional assumptions that introduce further complexity. In contrast, they opt for a very simple approach to the infection process with spatial dynamics. The process of adsorption of a virus $V$ by a susceptible tumoral cell $T$ (with rate $k_{1}$ ), and replication of $Y$ viruses that leave each infected cell $I$ (with rate $k_{2}$ ), is essentially described by the reactions

$$
V+T \stackrel{k_{1}}{\longrightarrow} I \stackrel{k_{2}}{\longrightarrow} Y \cdot V .
$$

Wodarz et al. study the behavior of an in vitro adenovirus in human embryonic kidney epithelial cells, experimentally and computationally, developing a simple model with two equations (see Eqs. (5) and (6) below), one for susceptible tumoral cells and one for infected cells. They make use of partial differential equations (PDEs) to model the virus-tumor system, because PDEs provide efficient information on the spatial and reactive mechanisms affecting the wave propagating fronts and PDEs can be used to compute their speeds.

The model by Wodarz et al. [5] is a two-equation system that was derived from a three-equation model due to Nowak and May [20]. Including diffusion and logistic growth, the Nowak-May model is

$$
\begin{aligned}
\frac{\partial[V](r, t)}{\partial t}= & D_{V} \frac{\partial^{2}[V](r, t)}{\partial r^{2}}+k_{2} Y[I](r, t) \\
& -k_{3}[V](r, t), \\
\frac{\partial[T](r, t)}{\partial t}= & D_{T} \frac{\partial^{2}[T](r, t)}{\partial r^{2}} \\
& +a[T](r, t)\left\{1-\frac{[I](r, t)+[T](r, t)}{k}\right\} \\
& -k_{1}[V](r, t)[T](r, t),
\end{aligned}
$$

$$
\begin{aligned}
\frac{\partial[I](r, t)}{\partial t}= & D_{I} \frac{\partial^{2}[I](r, t)}{\partial r^{2}}-k_{2}[I](r, t) \\
& +k_{1}[V](r, t)[T](r, t),
\end{aligned}
$$

where $[T],[I]$ and $[V]$ are the concentrations of susceptible tumoral cells, infected tumoral cells and viruses, respectively; $D_{T}, D_{I}$ and $D_{V}$ are their diffusion coefficients, $a$ the tumor growth rate, $k$ its carrying capacity, $k_{3}$ the decay rate of free viruses, $t$ the time and $r$ the radial coordinate (assuming radial symmetry, as explained in detail below). Some authors [20] have argued that, in some situations, it may be assumed that $\frac{\partial[\mathrm{V}]}{\partial t}=0$ and therefore, in homogeneous systems $\left(\frac{\partial^{2}[V]}{\partial r^{2}}=0\right)$, Eq. (2) implies that $[V](r, t)=\frac{k_{2} Y}{k_{3}}[I](r, t)$. However, this assumption (free virus in steady-state) could only be applied if the decay rate of virus $k_{3}$ is much larger than the decay rate of the infected cell population $k_{2}$ [20]. From these arguments, they obtain the two-equation system used by Wodarz et al. [5], namely

$$
\begin{aligned}
\frac{\partial[T](r, t)}{\partial t}= & D_{T} \frac{\partial^{2}[T](r, t)}{\partial r^{2}} \\
& +a[T](r, t)\left\{1-\frac{[I](r, t)+[T](r, t)}{k}\right\} \\
& -b[I](r, t)[T](r, t),
\end{aligned}
$$




$$
\begin{aligned}
\frac{\partial[I](r, t)}{\partial t}= & D_{I} \frac{\partial^{2}[I](r, t)}{\partial r^{2}}-k_{2}[I](r, t) \\
& +b[I](r, t)[T](r, t),
\end{aligned}
$$

where $b=\frac{k_{1} k_{2} Y}{k_{3}}$.

However, we find two drawbacks in the model (5)-(6) to explain our VSV-GBM system. First, Wodarz assumes $\frac{\partial[V]}{\partial t}=0$, and thus $[V] \propto[I]$. As said before, this may be valid when $k_{3} \gg k_{2}$ and in some non-spatial models [20] but this is in general not valid for the spatial propagation of virus infections. In such cases, at points located far away from the initially infected area, before the arrival of the infection front we have $[V]=0$, when the infection arrives $[V] \neq 0$, and after all viruses (and infected cells) have decayed, we have again $[V]=0$. Therefore, when dealing with spatial infection fronts we have $\frac{\partial[V]}{\partial t}=0$ only at early and late times, but $\frac{\partial[V]}{\partial t}>0$ when the first viruses arrive and $\frac{\partial[V]}{\partial t}<0$ after the passage of the infected front. Moreover, our experimental data (see "Parameter values" section) suggest that in our system $k_{3}$ is very close to $k_{2}$ and therefore, the assumption $k_{3} \gg k_{2}$ is not satisfied here either. Therefore, in contrast to Ref. [5], we cannot assume $\frac{\partial[V]}{\partial t}=0$, thus we deal with three differential equations (for viruses, susceptible tumoral cells, and infected tumoral cells).

Our second objection to the model (3)-(2) [and its simplification (5)-(6)] is that, according to the first reaction in Eq. (1), virus adsorption causes not only the same decrease in susceptible tumor cells [last term in Eq. (3)] as the increase in infected cells [last term in Eq. (4)], but also the same decrease in viruses. Thus a term $-k_{1}[V](r, t)[T](r, t)$ is missing in the right side of Eq. (2), in agreement with many previous works on virus infections [15-17, 21].

In the next section we develop a model which takes both points into account, as well as other important effects (namely, time-delay effects).

\section{Methods}

\section{Mathematical models}

Here we want to develop a simple, but complete model to understand the dynamics of a virus-tumor system. The theoretical model should be able to explain an in vitro experiment where a virus injected into the center of a tumor spreads through the tumor cell population in a basically two-dimensional geometry. Therefore, we can think of the virus-tumor system as formed by two fronts of propagation, which could be represented as two concentric circles if we assume radial symmetry. The diagram in Fig. 1 illustrates this idea. The outer circle represents the tumor cells, which spread to the outside through a nonspecific medium. The inner circle represents the viruses

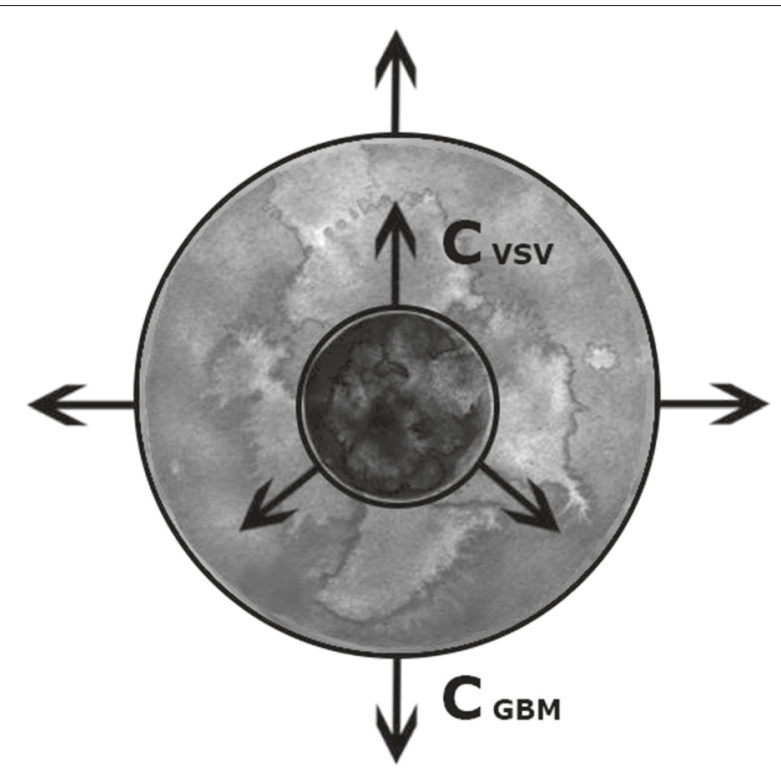

Fig. 1 Two circles representing the two propagation fronts of VSV and GBM. A front of tumor cells spreads radially (outer circle). After some time, viruses are inoculated at the center, and a virus front spreads (inner circle). If the inner circle expands faster than the outer one $\left(c_{V S V}>c_{G B M}\right)$, the viruses will eliminate the tumor

spreading within the tumor. Viruses diffuse through the medium before infecting tumor cells. When infected cells die, a new generation of viruses is created and the process begins anew.

The main idea and experimental laboratory data come from Ref. [9], where Wollmann et al. compare nine types of viruses with strong oncolytic potential and conclude that four of them, VSV included, would be worthy of more rigorous studies. Because in subsequent papers [11, 22] they worked with VSV and its recombinant variants or strains, we decided to focus solely on VSV and use these data as experimental basis.

Below we present three increasingly complete (and complicated) models.

\section{Model 1}

As a first approach, we adapt the model by Wodarz et al. [5] to the conditions in our VSV-GBM systems, i.e., we do not assume $\frac{d V}{d t}=0$, and therefore $[V]$ is not proportional to $[I]$ and we need to include the virus dynamics explicitly in the model.

Now the evolution of the virus-tumor system is described by

$$
\frac{\partial[V](r, t)}{\partial t}=D_{V S V} \frac{\partial^{2}[V](r, t)}{\partial r^{2}}+F(r, t),
$$




$$
\begin{aligned}
\frac{\partial[T](r, t)}{\partial t}= & D_{G B M} \frac{\partial^{2}[T](r, t)}{\partial r^{2}} \\
& +a[T](r, t)\left\{1-\frac{[I](r, t)+[T](r, t)}{k}\right\} \\
& -k_{1}[V](r, t)[T](r, t),
\end{aligned}
$$

$$
\frac{\partial[I](r, t)}{\partial t}=k_{1}[V](r, t)[T](r, t)-k_{2}[I](r, t) .
$$

The first equation describes the evolution of the virus population over time. The viruses can spread ruled by the diffusion coefficient $D_{V S V}$ and the Laplacian (or second space derivative). The function $F(r, t)$ in Eq. (7) incorporates all processes of infection, replication and death and is defined by

$$
\begin{aligned}
F(r, t)= & -k_{1}[V](r, t)[T](r, t) \\
& +k_{2} Y[I](r, t)-k_{3}[V](r, t) .
\end{aligned}
$$

Note that the first term was not included in the models by Nowak-May and Wodarz [Eq. (2)] (see our second objection in "Previous mathematical approaches" section).

Equation (8) describes the change in the number of tumor cells over time. Similarly to viruses, glioblastoma cells can also move, characterized by their own diffusion coefficient $D_{G B M}$.

Finally, Eq. (9) represents the evolution of infected tumoral cells. We assume that these cells do not move, in agreement Fig. 3D of Ref. [9], where the experiment shows how the infected cells (U-87 MG glioblastoma cells) initially introduced do not move through the host layer throughout the observation period.

\section{Model 2}

As we shall see in "Results and discussion" section, model 1 needs further improvements. In model 2 we take into account that infected tumoral cells do not die instantaneously, instead there is a time delay before the cell dies and releases the new progeny of viruses. We will denote this delay or eclipse time as $\tau$ and include it into the terms related to the death of infected cells. Thus infected cells will not die proportionally to the density of infected cells at the present time, $k_{2}[I](r, t)$, but proportionally to the density of infected cells at a previous instant $t-\tau, k_{2}[I](r, t-\tau)$, to properly include this time delay effect on the decay process. It has been shown that the term $-k_{2}[I](r, t-\tau)$ agrees well with experimental data in a different context (infections of non-tumor cells) [23]. Other reaction-diffusion models do also apply $t-\tau$, although in an alternative way [24, 25]. The differences between their approach and ours is analyzed in Ref. [23].
Therefore, when introducing the delay in the death of infected cells, Eqs. (9) and (10) are modified directly and Eq. (7) changes because the function $F(r, t)$, Eq. (14), is also modified. We do not modify the growth term in Eq. (8) because the reproduction of tumoral cells depends on the total number of tumor cells (infected and susceptible) at that precise instant $t$. So, we consider the model

$$
\begin{aligned}
\frac{\partial[V](r, t)}{\partial t}= & D_{V S V} \frac{\partial^{2}[V](r, t)}{\partial r^{2}}+F(r, t) \\
\frac{\partial[T](r, t)}{\partial t}= & D_{G B M} \frac{\partial^{2}[T](r, t)}{\partial r^{2}} \\
& +a[T](r, t)\left\{1-\frac{[I](r, t)+[T](r, t)}{k}\right\} \\
& -k_{1}[V](r, t)[T](r, t),
\end{aligned}
$$

$$
\frac{\partial[I](r, t)}{\partial t}=k_{1}[V](r, t)[T](r, t)-k_{2}[I](r, t-\tau),
$$

where now

$$
\begin{aligned}
F(r, t)= & -k_{1}[V](r, t)[T](r, t) \\
& +k_{2} Y[I](r, t-\tau)-k_{3}[V](r, t) .
\end{aligned}
$$

This second model is, actually, an approximation of our next model (see Model 3 below).

\section{Model 3}

Model 2 takes into account a delay time in the reactive process $I \rightarrow Y \cdot V$, but here we shall see that the delay time also has a very important diffusive effect. The diffusion dynamics of the virus concentration in Eq. (11) is Fickian, which means that it does not take into account the effect of the time delay $\tau$. In year 2002 it was shown [26] that it is very important to take into account that $\tau$ is the time interval during which a virus does not move in space (because it is inside an infected cell), thus the delay time should affect the model by slowing down the spread of viruses. Therefore it is necessary to incorporate also this effect to reach a realistic model. For this reason, Eq. (11) must be replaced by an equation with second-order terms to include this diffusive time-delay effect $[17,26,27]$.

Thus, finally we describe the spatial-time dynamics of the whole system with the following equations:

$$
\begin{aligned}
\frac{\partial[V](r, t)}{\partial t} & +\frac{\tau}{2} \frac{\partial^{2}[V](r, t)}{\partial t^{2}}=D_{V S V} \frac{\partial^{2}[V](r, t)}{\partial r^{2}} \\
& +F(r, t)+\left.\frac{\tau}{2} \frac{\partial F(r, t)}{\partial t}\right|_{g}
\end{aligned}
$$




$$
\begin{aligned}
\frac{\partial[T](r, t)}{\partial t}= & D_{G B M} \frac{\partial^{2}[T](r, t)}{\partial r^{2}} \\
& +a[T](r, t)\left\{1-\frac{[I](r, t)+[T](r, t)}{k}\right\} \\
& -k_{1}[V](r, t)[T](r, t),
\end{aligned}
$$

$$
\frac{\partial[I](r, t)}{\partial t}=k_{1}[V](r, t)[T](r, t)-k_{2}[I](r, t-\tau),
$$

where the terms proportional to $\tau$ in Eq. (15) are the new, second-order terms. A self-contained derivation of Eq. (15) can be found in Ref. [23], Appendix A.

In Eq. (15) $F(r, t)$ is again given by Eq. (14), and Eqs. (12) and (13) from model 2 remain unchanged (Eqs. (16) and (17), respectively).

Note that $F(r, t)$ can be understood as the variation of $[\mathrm{V}]$ over time due to all reactive processes, but not to diffusive processes, i.e. $F(r, t)=\left.\frac{\partial[V](r, t)}{\partial t}\right|_{g}$. This allows the proper calculation of the first time derivative as $[17,27]$

$$
\begin{aligned}
\left.\frac{\partial F(r, t)}{\partial t}\right|_{g}= & -k_{1} F(r, t)[T](r, t)-k_{1}[V](r, t) \frac{\partial[T](r, t)}{\partial t} \\
& +k_{2} Y \frac{\partial[I](r, t-\tau)}{\partial t}-k_{3} F(r, t) .
\end{aligned}
$$

For systems in which the infected cells diffuse appreciably (not our case, see the last paragraph in the model 1 section), an age-structure model with this additional diffusive-delay effect has been proposed by Gourley and Kuang in Ref. [24], p. 558.

In the equation describing the virus dynamics, Eq. (15), we include corrections only up to second order [17, 27]. It has been shown in previous work [26] that the divergence between second-order approximation and full timedelayed equations is small, and thus we can exclude terms of higher orders.

\section{Front speeds \\ Virus front}

Using models 1-3 above, we look for realistic travellingwave speeds for both the propagation front of viruses (inner front, Fig. 1) and the propagation front of tumor cells (outer front, Fig. 1). Finding the propagation speeds will allow us to compare to the in vitro experiments in order to validate our approach.

In all models $1-3$, we can transform the problem into a single-variable system by using the co-moving coordinate $z=r-c t$. Like in previous works [15, 26], we assume the concentration of the three populations at the leading edge of the moving front $(z \longrightarrow \infty)$ can be written as $[T]=k-\epsilon_{T} \cdot \exp (-\lambda z),[I]=\epsilon_{I} \cdot \exp (-\lambda z)$ and $[V]=$
$\epsilon_{V} \cdot \exp (-\lambda z)$, thus we assume that tumoral cells are nearly at maximum concentration at large distances from the inoculation point of the viruses, while viruses and infected cells are barely present. We make use of this transformation because beyond the edge of the front of infected cells and viruses, there is only a continuous medium of tumor cells. For non-trivial solutions to exist, the determinant of the matrix corresponding to the linearized model must be zero. The characteristic equations for models 1,2 and 3 are, respectively,

$$
\begin{gathered}
\left(\lambda c+k_{2}\right)\left(\lambda c-D_{V S V} \lambda^{2}+k k_{1}+k_{3}\right) \\
-k k_{1} k_{2} Y=0 \\
\left(\lambda c+k_{2} \mathrm{e}^{-\lambda c \tau}\right)\left(\lambda c-D_{V S V} \lambda^{2}+k k_{1}+k_{3}\right) \\
-k k_{1} k_{2} Y \mathrm{e}^{-\lambda c \tau}=0, \\
\left(\lambda c+k_{2} \mathrm{e}^{-\lambda c \tau}\right)\left[\lambda c-D_{V S V} \lambda^{2}+k k_{1}+k_{3}\right. \\
\left.+\frac{\tau}{2}\left(\lambda^{2} c^{2}-k^{2} k_{1}^{2}-2 k k_{1} k_{3}-k_{3}^{2}\right)\right] \\
-k k_{1} k_{2} Y \mathrm{e}^{-\lambda c \tau}\left[1+\frac{\tau}{2}\left(\lambda c-k k_{1}-k_{3}\right)\right]=0 .
\end{gathered}
$$

According to marginal stability analysis [28], the propagation front moves with the minimum possible speed. Therefore,

$$
c_{V S V}=\min _{\lambda>0}[c(\lambda)],
$$

where $c(\lambda)$ is given implicitly by Eqs. (19), (20) and (21). From Eq. (22) we can numerically estimate the speed of VSV infection.

The resulting propagation speeds for models $1-3$ will be calculated and plotted in "Results and discussion" section.

We also solve the third model by numerical integration and find the front speed from the position of the virus front wave in a successive steps of time.

\section{Glioblastoma front}

Under the hypothesis of two propagation fronts, as shown in Fig. 1, the outermost front would correspond the tumor cells, $[T]$ (GBM in our case of study). In the conditions near this front, all models can be greatly simplified since here the populations of viruses and infected cells are zero (see the outer circle in Fig. 1 for a better understanding), so $[V](r, t)=0$ and $[I](r, t)=0$. Hence, it is only necessary to work with the equation for the tumoral cells, Eq. (16) for example, but remembering that $[V](r, t)=$ $[I](r, t)=0$,

$$
\begin{aligned}
\frac{\partial[T](r, t)}{\partial t}= & D_{G B M} \frac{\partial^{2}[T](r, t)}{\partial r^{2}} \\
& +a[T](r, t)\left\{1-\frac{[T](r, t)}{k}\right\} .
\end{aligned}
$$


At the leading edge of this front, we assume that $[T](r, t)=\epsilon_{T} \cdot \exp (-\lambda z)$, and after some algebra we easily obtain the speed of the glioblastoma front,

$$
c_{G B M}=2 \sqrt{D_{G B M} a},
$$

where $D_{G B M}$ is the glioblastoma diffusion coefficient and $a$ the growth rate, both estimated in the next subsection. Note that Eq. (24) is the well-known Fisher propagation speed [29]. Some recent extensions have been proposed $[6,30]$, but they are not necessary for the purposes of the present paper.

\section{Parameter values}

We estimate most of our parameters from in vitro experiments on VSV applied to GBM [9, 11, 22]. The parameters that we could not draw from such experiments have been obtained from other rigorous studies on VSV or glioblastoma.

We use two different values of $D_{V S V}$ because the diffusion coefficient of VSV has not been measured in gliomas. The only value of VSV available (measured in an specific water solution) is $D_{V S V}=8.37 \cdot 10^{-5} \mathrm{~cm}^{2} / \mathrm{h}$ [31]. Another value measured in agar of VSV-similar viruses is $D_{V S V}=1.44 \cdot 10^{-4} \mathrm{~cm}^{2} / \mathrm{h}[17]$.

Concerning $D_{G B M}$, Stein et al. [32] performed an in vitro experiment in which a GBM tumor spheroid is implanted into a collagen gel. The diffusion coefficient is measured by tracking individual cells on the first day, calculating their motion and averaging over many cells. Stein and coworkers measure diffusion coefficients in the radial and angular directions, which lead to the value $D_{G B M}=3.75$. $10^{-6} \mathrm{~cm}^{2} / \mathrm{h}[6]$.

Besides spreading, the number of cells also increases. The parameter $a$ is the corresponding proliferation rate. In vitro measurements provide ample scope for this parameter, $0.04<a<0.3$ day $^{-1}$ [33], and similarly in vivo studies yield $0.01<a<0.14$ day $^{-1}$ [34].

The saturation cell density, $k$, measures the maximum concentration of tumor cells (susceptible and infected) per unit volume that the system can support, and its usual value is $k=10^{6}$ cells $/ \mathrm{cm}^{3}$ (e.g.,Refs. [35, 36]).

We next analyze the rest of parameters, which are calculated from the experimental studies by Wollmann et al. $[9,11,22]$.

The yield or burst size $Y$ represents the total amount of viruses produced by the death of a single infected cell. There is no accurate numerical value calculated for the case of VSV infecting GBM. However, by studying Fig. 4 in Ref. [11] we can obtain an estimation. The burst size can be understood as the ratio between the maximum and initial number of viruses, i.e. $Y=\frac{V_{\max }}{V_{0}}$. From that figure, $V_{0}$ is between $10-100 \mathrm{PFU} / \mathrm{ml}$ (last two plots in Fig. 4 in [11]) and $V_{\max }$ between $10^{8}-10^{9} \mathrm{PFU} / \mathrm{ml}$ (the maximum is reached between 1 and 2 days post infection), so we conclude that $10^{6}<Y<10^{8}$. This also agrees with the value measured in Ref. [37], although in that case VSV infects BHK-21 cells (not GBM cells).

We have seen that there is a time lapse between a cell being infected by a virus and that cell dying (and therefore, adding more viruses to the system). This time lapse is called the delay time, $\tau$. It plays a main role in the virus propagation speed, but has not been accurately measured. From the in vitro experiments described in Ref. [9] we can try to estimate the value of $\tau$. On one hand, we know that the death of infected cells begins about 6 hours post infection (hpi) of the virus to susceptible tumoral cells. We also know that infected cells can be seen as early as 4 hpi (they are tracked down using GFP fluorescence). From both data, we conclude that viruses leave infected cells at least $2 \mathrm{~h}$ after infection. On the other hand, in a different experiment infected cells are added directly (rather than infecting viruses) and new infected cells were detected after $12 \mathrm{~h}$. This period includes the time needed for the viruses to multiply within the infected cells, leave the cell and infect new tumoral cells. So we can also assume that $\tau$ must be lower than $12 \mathrm{~h}$. In summary, we will work with the range $2<\tau<12 \mathrm{~h}$.

The adsorption rate, $k_{1}$, describes the efficacy of the whole infection process (rate of virus entry and probability of successful infection). The value of $k_{1}$ could be measured in an experiment where the reproduction of viruses and host cells were prevented. Such an experiment has been performed for other viruses [38] but not for VSV infecting GBM. Since we do not have the ideal conditions in the experiments cited before $[9,11,22]$, we will use the earliest data post-inoculation available in the experimental data in Ref. [11] to minimize the effect of reproduction and thus obtain the best possible estimation for $k_{1}$.

Equations (7) and (8) are simplified in the absence of reproduction and natural death, and when the population is studied as a whole (i.e. ignoring diffusion terms) we have

$$
\frac{d[V](t)}{d t}=\frac{d[T](t)}{d t}=-k_{1}[V](t)[T](t) .
$$

Obviously, integrating we get $[T](t)=[V](t)+\xi$, where $\xi$ is the constant of integration. Note that $\xi$ is the difference between the concentrations of tumor cells and viruses. In order to estimate $k_{1}$, we can rewrite the previous Eq. (25) as $\frac{d[T](t)}{d t}=-k_{1}[T](t)([T](t)-\xi)$ and making the necessary algebra we obtain the final formula for calculating the adsorption rate,

$$
k_{1}=\frac{1}{\xi\left(t-t_{0}\right)}\left[\ln \left(\frac{T}{T-\xi}\right)-\ln \left(\frac{T_{0}}{T_{0}-\xi}\right)\right] .
$$

It is difficult to know the exact concentration of cells at the beginning of the experiment or at certain time $t$, because only relative concentrations were reported. However, extrapolating data provided in the previous cited 
papers by Wollmann et al. (Fig. 3C Control in [11], bar G/GFP), we believe it is correct to assume that the values of initial tumor cells lie in the range $T_{0}=10^{6}-10^{8}$ cells $/ \mathrm{cm}^{3}$, and that $T=0.65 T_{0}$ cells $/ \mathrm{cm}^{3}, t-t_{0}=36$ $\mathrm{h}$. This allows the calculation of the adsorption rate, as $5 \cdot 10^{-10}<k_{1}<5 \cdot 10^{-8} \mathrm{~cm}^{3} / \mathrm{h}$. This is a rather wide range, but we show in "Effect of $k_{1}$ and $Y$ " section that $k_{1}$ (as well as $Y$ ) does not overly affect the propagation front speed of VSV.

Finally, parameters $k_{2}$ and $k_{3}$ correspond to the rates of death of infected cells and virus, respectively. Therefore, the average life-time of an infected cell and a virus are $1 / k_{2}$ and $1 / k_{3}$, respectively.

The rate of death of infected cells $k_{2}$ could be also understood as the growth of viruses. Thus, for $t<\tau$ no new virus are seen in the corresponding experiment (because no infected cell has died yet), but for $t \geq \tau$ the infected cells start to die ruled by $d I=-k_{2} I_{0} d t$. The death of each infected cell produces $Y$ virus, thus $d V=-Y d I=$ $k_{2} Y I_{0} d t=k_{2} V_{\max } d t$. Integrating, we get $k_{2}=\frac{V_{\max }-V_{0}}{\Delta t \cdot V_{\max }} \approx$ $\frac{1}{\Delta t}=\frac{1}{t^{*}-\tau}$, where $t^{*}$ represents the time when the virus population reaches its maximum. According to Fig. 4B in Ref. [11], experimental data (labeled as VSV-G/GFP) show that the maximum is reached at $t^{*}=(48 \pm 12) \mathrm{h}$. Nevertheless, the final result of $k_{2}$ will depend on $\tau$ and we have a range rather than a single value for $\tau$ (see above). Note, however, that for model 1 there is no time delay, so $k_{2}$ is calculated straightforwardly as the inverse of time $t^{*}$ at which the concentration of viruses reaches its maximum, $k_{2}=\frac{1}{t^{*}} \mathrm{~h}^{-1}$. Models 2 and 3 are dealt with in "Results and discussion" section.

The evolution of the viruses over time in an environment where they die but cannot reproduce is ruled by $d V=-k_{3} V d t$. Through simple integration we get $V(t)=V_{0} \exp \left[-k_{3}\left(t-t_{0}\right)\right]$. In the same experiment as before, Fig. 4B in Ref. [11], we now have two cases where these conditions are exactly reproduced (because VSV-dG-GFP and VSV-dG-RFP are replication-restricted virus variants, so they basically die). We can estimate both values of $k_{3}$ from the experimental data, namely $V(t=24 \mathrm{~h})=30 \mathrm{PFU} / \mathrm{cm}^{3}, V(t=48 \mathrm{~h})=20 \mathrm{PFU} / \mathrm{cm}^{3}$ and $V(t=72 \mathrm{~h})=8 \mathrm{PFU} / \mathrm{cm}^{3}$ for the mutant dG-GFP and $V(t=24 \mathrm{~h})=12 \mathrm{PFU} / \mathrm{cm}^{3}, V(t=48 \mathrm{~h})=8$ $\mathrm{PFU} / \mathrm{cm}^{3}$ and $V(t=72 \mathrm{~h})=6 \mathrm{PFU} / \mathrm{cm}^{3}$ for dG-RFP. Performing linear fits to $\ln V$ versus $t$, we obtain that $0.014<$ $k_{3}<0.028 \mathrm{~h}^{-1}$.

\section{Results and discussion}

\section{GBM and VSV front speeds: theory versus experiment}

Our main objective is to obtain realistic values for the propagation speeds in an in vitro virus-tumor system, providing positive results from a biophysical point of view for the realization of these treatments.
In "Methods" section we have described three possible models for our VSV-GBM system and the necessary experimental parameter values. Here we present the speeds predicted by these models.

The case of tumor expansion has a single, simple solution for all models, Eq. (24), since the infection does not play a role here. Substituting the values of $D_{G B M}$ and $a$ we obtain that $c_{G B M}=2.5 \cdot 10^{-4} \mathrm{~cm} / \mathrm{h}$, with $a=0.1$ day $^{-1}$, which we think is a reasonable mean value. Indeed, the range of measurements of the proliferation rate is $0.01<a<0.3 \mathrm{day}^{-1}$, which yields a range of speeds $7.9 \cdot 10^{-5}<c_{G B M}<4.33 \cdot 10^{-4} \mathrm{~cm} / \mathrm{h}$ ). Stein and coworkers measured an experimental in vitro speed range of $2.37 \cdot 10^{-4}<c_{G B M}<5.54 \cdot 10^{-4} \mathrm{~cm} / \mathrm{h}$ [33], which is consistent with our model, despite the simplicity of Eq. (24).

The case of the virus front is less straightforward. As we have already discussed in "Parameter values" section, a very important but not strictly well-measured parameter is the delay time $\tau$. Therefore, the speed results have been calculated in terms of this parameter, $c(\tau)$. The death rate of infected cells $k_{2}$ also changes, because it depends directly on $\tau$.

The infection front speed, $c_{V S V}$, can be seen in Fig. 2 . For each of the 3 models we have plotted the results from typical parameter values (bold lines). To compute these results we have chosen the parameter values that seem to be the most representative and accepted for this experiment: average values of $k_{2}$ and $k_{3}$, the value of $D_{V S V}$ calculated for VSV in an specific water solution and the larger values of $k_{1}$ and $Y$. However we have also computed $c_{V S V}$ by varying each of the parameters of Eqs. (19)-(21), with the exception of $k$ because $k=10^{6}$ cells $/ \mathrm{cm}^{3}$ is a widely accepted value in research papers (see "Parameter values" section). In Fig. 2 we include the upper and lower bounds for the front speed obtained, for each of the 3 models, from the experimental parameter ranges (parameter values are specified at the caption).

The hatched area in Fig. 2 corresponds to the experimental values of VSV speed estimated from the in vitro experiment by Wollmann et al. in Ref. [9], Fig. 3A.

Dotted lines correspond to the analytical results to model 1, Eqs. (7)-(10), i.e. the classical model adapted from the equations in Ref. [5]. Obviously they are horizontal lines, since they do not depend on $\tau$. As we can see in Fig. 2, model 1 yields speeds much faster than the experimental observations. The curves are the numerical results from our time-delayed reaction-diffusion models. Dashed curves correspond to model 2, given by Eqs. (11 )(14). We see that just by taking into account the eclipse or delay time on the death of infected cells, we obtain much better results as compared with experimental velocities, although not enough to satisfactorily explain the data (the 


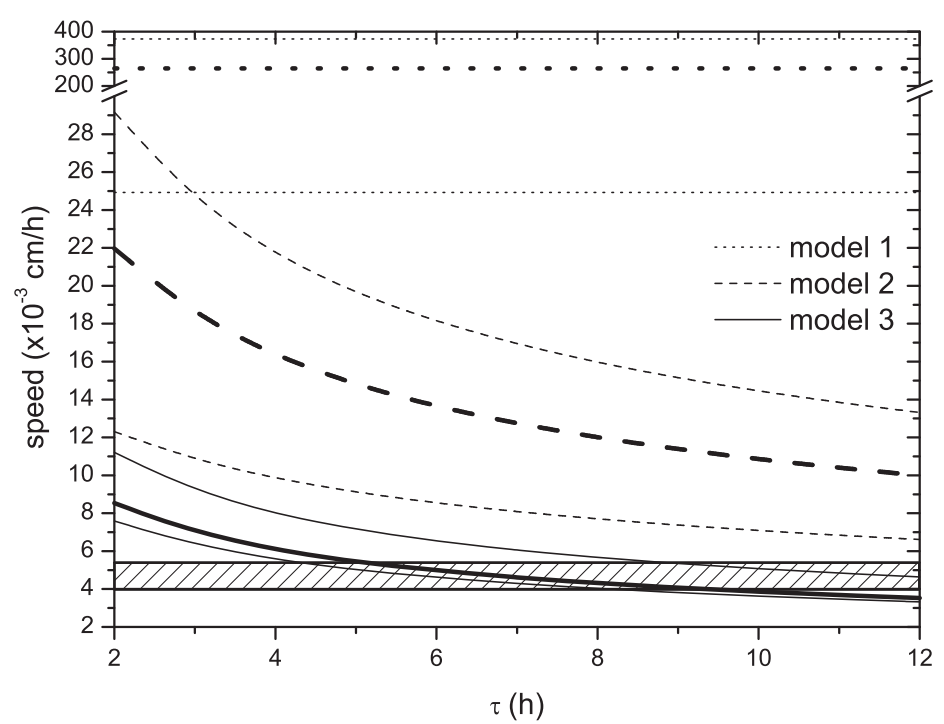

Fig. 2 VSV front propagation speed as a function of the delay time $\tau$, for model 1 (dotted lines), model 2 (dashed curves) and model 3 (solid curves). The hatched area shows the experimental in vitro VSV front speed [9]. Upper bounds are computed from: $k_{1}=5 \cdot 10^{-8} \mathrm{~cm}^{3} / \mathrm{h}, k_{2}=\frac{1}{36-\tau} \mathrm{h}^{-1}$ $\left(k_{2}=\frac{1}{36} \mathrm{~h}^{-1}\right.$ for model 1$), k_{3}=0.014 \mathrm{~h}^{-1}, Y=10^{8}$ and DVVV $=1.44 \cdot 10^{-4} \mathrm{~cm}^{2} / \mathrm{h}$. Lower bounds are computed from: $\mathrm{k}_{1}=5 \cdot 10^{-10} \mathrm{~cm}^{3} / \mathrm{h}$, $k_{2}=\frac{1}{60-\tau} h^{-1}\left(k_{2}=\frac{1}{60} h^{-1}\right.$ for model 1$), k_{3}=0.028 \mathrm{~h}^{-1}, Y=10^{6}$ and $D_{V S V}=8.37 \cdot 10^{-5} \mathrm{~cm}^{2} / \mathrm{h}$. The results from typical values (bold lines) are computed from: $k_{1}=5 \cdot 10^{-8} \mathrm{~cm}^{3} / \mathrm{h}, k_{2}=\frac{1}{48-\tau} \mathrm{h}^{-1}\left(\mathrm{k}_{2}=\frac{1}{48} \mathrm{~h}^{-1}\right.$ for model 1$), \mathrm{k}_{3}=0.02 \mathrm{~h}^{-1}, Y=10^{8}$ and $D_{\text {VSV }}=8.37 \cdot 10^{-5} \mathrm{~cm}^{2} / \mathrm{h}$. In all the cases $k=10^{6} \mathrm{cells} / \mathrm{cm}^{3}$

minimum bound of model 2 in Fig. 2 is above the hatched area). Finally, solid curves in Fig. 2 correspond to model 3 (please recall that this is extremely close to the full timedelayed equation, see "Methods" section). The equations for this main model, Eqs. (15)-(18), when considering typical parameter values, produce results that agree with the experimental data within a range of $\tau$ between 5.0 and $9.3 \mathrm{~h}$.

According to our best description (model 3), the entire range of speed $c_{V S V}$ in Fig. 2 is an order of magnitude faster than the speed of propagation of glioblastoma, $c_{G B M}$, (see above). Therefore the virus front could theoretically reach the tumoral front and infect it all. We stress that this is a model appropriate for in vitro experiments, whereas in vivo more complex models will be necessary (as discussed below).

In Fig. 3 we show snapshots of the viruses and infected cells profiles as functions of the radial axis, computed from the computational simulations at three time instants. The simulations have been performed by numerical integration of model 3, which is biologically more realistic and produces results in agreement with the experimental data (see Fig. 2). We use the typical parameter values used in Fig. 2 (bold lines, see caption for the values). We can see in Fig. 3 that both propagation fronts advance at the same speed and with regular shapes.

From the profiles we can see that the number of infected cells grows rapidly, then there is a plateau of infected cells (as a result of the time delay $\tau$ before any infected cell dies), and then decay at a rate $k_{2}$. The virus profiles show an abrupt rise when infected cells start dying (end of the plateau of infected cells) and then keep rising up to a peak. Behind this peak, the virus death term $k_{3}$ predominates over the virus production, and the number of viruses decay. Although Fig. 3 seems to indicate that the front of infected cells appears prior to the virus front, the opposite happens (this can be appreciated by enlarging the vertical scale).

From these simulations we can calculate the front speed by tracking the position of the edge of the front of the virus at successive steps of time. A simple space vs time data is generated and then, the front speed is directly the slope. From the simulations (parameter values are the same than typical values in Fig. 2 with $\tau=6 \mathrm{~h}$ ) we find a front speed of $4.829 \cdot 10^{-3} \mathrm{~cm} / \mathrm{h}$. The relative error between the simulations and the analytic speed $\left[c_{V S V}=4.853 \cdot 10^{-3} \mathrm{~cm} / \mathrm{s}\right.$, from Eqs. (21)-(22)] is only about $0.5 \%$.

An alternative way to know the front propagation speed from Fig. 3 is the plateau of infected cells. Its width is directly related with the time delay $\tau$ and the infection front speed as width $=\tau \cdot c$. Then, the result for the speed is $(0.53858-0.51317) \mathrm{cm} / 6 \mathrm{~h}=4.735 \cdot 10^{-3} \mathrm{~cm} / \mathrm{h}($ distances for $t=108 \mathrm{~h}$ ), and the relative error (compared with the analytical results with same parameter values than the simulations) is lower than $2.5 \%\left(c_{V S V}=4.853\right.$. $\left.10^{-3} \mathrm{~cm} / \mathrm{s}\right)$. 


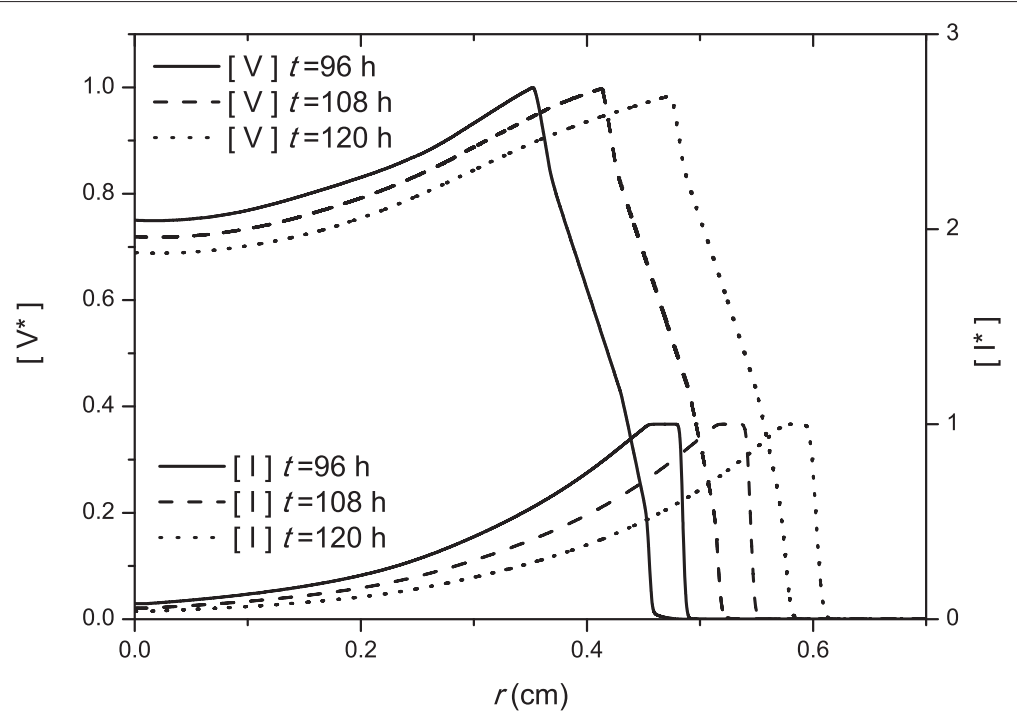

Fig. 3 Radial profiles of $\left[\mathrm{V}^{*}\right]$ and $\left[{ }^{*}\right]$ at three different times for model 3 . The labels of $V^{*}$ and $l^{*}$ stand for the units used, defined as $\frac{[\mathrm{V}]}{[\mathrm{V}]_{\max }}$ and $\frac{[\mathrm{l}}{[]_{\max }}$, respectively. The profiles are computed from numerical integration

\section{Effect of $k_{1}$ and $Y$}

In "Parameter values" section we have estimated the values of the parameters used in our mathematical models. Some of them, e.g. $D_{V S V}, D_{G B M}$ and $k$, have well-defined values, which are taken from the references indicated in the text. The delay time $\tau$ plays a very important role and therefore we have found the front propagation speed as a function of this parameter (remember that $k_{2}=\frac{1}{48-\tau}$, so we could add $k_{2}$ to this argument). Other parameters like $a$ and $k_{3}$ have a range of possible values, albeit a narrow one, and as such we use the mean value, or that usually accepted by other sources. Lastly, parameters $Y$ and $k_{1}$ have very wide ranges, spanning several orders of magnitude, but as we shall show below, they do not have an important effect on the virus front speed.

In Fig. 4 the speed of VSV is calculated from model 2 (Eqs. (11)-(14)) and model 3 (Eqs. (15)-(18)). Setting the typical parameter values previously used in Fig. 2 (bold curves) and Fig. 3 for $D_{V S V}, D_{G B M}, k, k_{3}$ and the average value $\tau=8 \mathrm{~h}$ (so $k_{2}=1 / 40 \mathrm{~h}^{-1}$ ), which yields results consistent with the range of experimental speeds (Fig. 2), we have varied the values of $Y$ and $k_{1}$ for each of both models.

In model 2 (upper curves in Fig. 4) the speed dependence on $Y$ and $k_{1}$ is fairly important. Indeed, by increasing these variables by two orders of magnitude, the speed increases on average by 25 and $18 \%$, respectively. However, looking at the best approach, model 3 (lower curves), we note that the speed increases only by 3 and $2 \%$ for $Y$ and $k_{1}$, respectively.

Therefore, model 3 has little dependence on the parameters $Y$ and $k_{1}$ and the delay time is the most important parameter (Fig. 2). In contrast, model 2 depends more directly on both parameters, although $\tau$ still remains the crucial one (compare the change of the speed in Fig. 2 with those in Fig. 4 for model 2). To obtain a speed of virus propagation similar to the observed data $\left(c \approx 5 \cdot 10^{-3}\right.$ $\mathrm{cm} / \mathrm{h}$ ) with model 2 , we should modify $Y$ and $k_{1}$ out of the experimental ranges. Indeed, their values should be about $Y=10^{4}$ or $k_{1}=5 \cdot 10^{-12} \mathrm{~cm}^{3} / \mathrm{h}$. Therefore, we could get a speed in agreement with the experimental data, but only using unrealistic parameter values, which do not correspond to VSV. This is further proof that our final model 3, the time-delayed reaction-diffusion set of equations, is a good mathematical tool to explain this kind of virus-tumor biological systems.

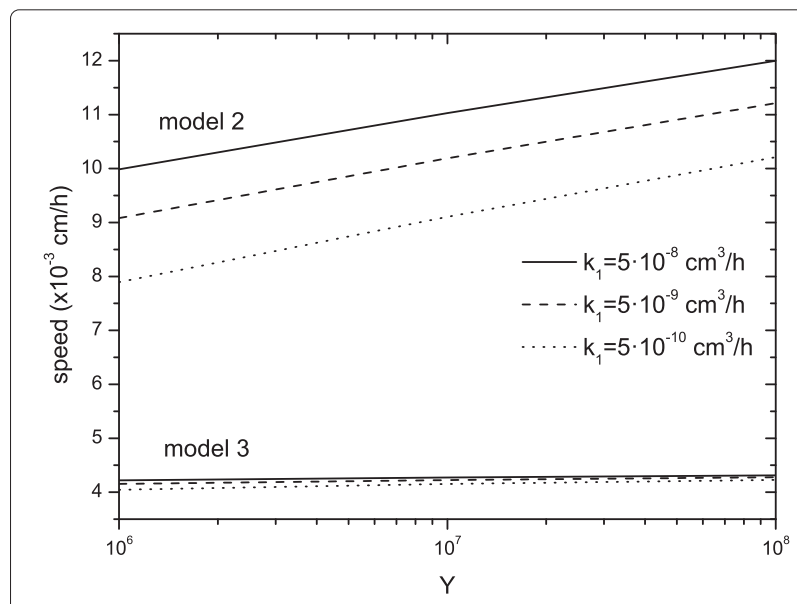

Fig. 4 VSV invasion speed on GBM for various values of $Y$ and $k_{1}$. The other parameter values are $k=10^{6} \mathrm{~cm}^{-3}, k_{2}=\frac{1}{40} \mathrm{~h}^{-1}, \tau=8 \mathrm{~h}$ and $k_{3}=0.02 h^{-1}$. Model 3 proves that neither $Y$ nor $k_{1}$ affect much the speed of the front 


\section{Conclusions}

A simple set of time-delayed equations have been built to understand the dynamics of a virus-tumor system. We have improved a previous model with new ideas and carefully incorporated experimental results (especially Ref. [9]). Figure 2 proofs that our best framework (model 3) is in reasonable agreement with the experimental data. Furthermore, the figure shows that neither model 1 nor model 2 can explain the experimental data. So it is absolutely necessary to add the second-order terms proportional to $\tau$ in Eq. (15) to properly include the time-delay effect.

We have shown that the delay time $\tau$ is the crucial parameter in our models (even when compared to other parameters that are strongly unknown, such as $k_{1}$ and $Y$ ). As we could have expected, as $\tau$ increases, the speed of the virus front decreases, because viruses spend more time inside the cell, and therefore at rest. In spite of being of utmost importance, the role of the delay or eclipse time has not been taken into account in previous models of virus treatment of tumors $[5,18,19]$.

We have found that our new model can satisfactorily predict the front speed for the lytic action of oncolytic VSV on glioblastoma observed in vitro. But this is only a first step towards a deep biophysical understanding of the principles of virus-tumor space-time spread in a complex system. This model could be extended to be applied to in vivo experiments where, among other effects, the immune response should be also included in the model because it may play a significant role regulating the efficacy of the therapy. In particular, it seems that there is currently no agreement about which approach is better in oncolytic therapy, whether to modify oncolytic viruses to obtain the maximum antitumoral immune response [39], to transiently suppress the immune response [40], or to use a combination of both [40]; future appropriate modeling of the three scenarios might help in tackling this controversy from a different perspective.

In this paper we have focused on GBMs because of the experimental data available, but our model could apply also to many non-diffusive cancers, for which viral therapy is a promising approach $[18,19,41]$, since the reactiondiffusion equations for the viruses [Eqs. (15)-(18)] will still be valid, even though in such cases tumor cells will not diffuse. Thus, we provide a basis that can be applied in the near future to realistically simulate in vivo virus treatments of several cancers.

\section{Reviewers' comments}

\section{Reviewer's report 1}

Yang Kuang, Arizona State University, United States of America

Reviewer comments: The paper is mostly well written with only a few places where I can suggest the authors to consider adding more details or be aware of alternative explanations.

1 : The authors made a valid point that $\frac{\partial[V]}{\partial t}$ is not always close to 0 . However, a routine argument used in the mathematical modeling community is the quasi-state-steady approximation. This argument suggests that due to virus' fast dynamics (virus reproduces probably in less than one hour once the first virus reproduced), over the longer period tumor cell growth time (of days), on average, the total virus amount changes at a rate far less than the maximum possible rate when all viruses reproduce at the maximum rate. Mathematically, one can show this quasisteady-state level can be approximated by setting $\frac{\partial[V]}{\partial t}=0$ and solving $V$ in terms of other variables. 2: A better reference in the virus modeling context for the need of adding the virus loss term $-k_{1}[V](r, t)[T](r, t)$ may be E. Beretta and Y. Kuang: Modeling and analysis of a marine bacteriophage infection. Math. Biosc. 149, 5776(1998), where each and every term is carefully explained in the context of biology. 3: The justification for the Eq. (15) is mathematically simple, but mechanistically very ad hoc and difficult to follow. A possible alternative way to modeling the delay dependence of the diffusive action is to assign virus an age. A good reference on this approach is S. A. Gourley and Y. Kuang: A Delay Reaction-Diffusion Model of the Spread of Bacteriophage Infection, SIAM J. Appl. Math., 65, 50566(2005). 4: I think readers will benefit if the authors can provide more about the data nature and even a figure which may suggest that the VSV front is as described in Fig. 2. The authors may take a look of our recent work on in vitro GBM modeling and wave speed estimation to see how we handled this. Tracy L. Stepien, Erica M. Rutter, and Yang Kuang, 2015. A datamotivated density-dependent diffusion model of in vitro glioblastoma growth, Math. Biosc. Eng., 12, 11571172.

Authors' response: We want to thank Dr. Y. Kuang for his revision of our manuscript and the suggestions provided to make it more complete and comprehensive. We answer each of his four comments separately below:

1. The quasi-state-steady approximation is truly widely used in mathematical modeling. It implies that the virus dies in a very short time, and the rate of the virus producing infected cells is short enough not to create a great amount of viruses. Mathematically, this means that, $k_{3} \gg k_{2}$ [20]. This condition is not fulfilled in our VSV-GBM system, where $k_{3} \approx k_{2}$. As a result, we consider that, in such a system, it is better to develop our model and perform the calculation with all three equations. We explain this before Eqs. (5) and (6).

2. We have added the relevant reference suggested at the end of "Previous mathematical approaches" section.

3. The justification of Eq. (15) is described in more detail in Ref. [23], Appendix A. We mention this below Eq. (17). 
We also cite [below Eq. (18)] the interesting reference suggested, which applies to systems in which infected cells diffuse (not our case).

4. A new figure (Fig. 3) has been added to the paper showing the evolution in space and time of the concentration of virus and infected cell populations. We have computed these profiles through numerical integration and they now provide a new source from where to calculate the front speed for model 3. We see that this new value agrees with the experimental data found in Wollmann et al. experiments and with our analytical results. Readers will probably benefit from this new approach in order to completely understand the significance of our new equations and the good agreement between theoretical and experimental data. So, we specially appreciate the advice (from both referees) to include this kind of results.

\section{Reviewer's report 2}

Georg Luebeck, Fred Hutchinson Cancer Research Center, United States of America

Reviewer comments: The mathematical framework presented by de Rioja et al. for oncolytic infection of GBM cells by the VSV virus and its impact on tumor growth in culture builds upon previous modeling. The authors show, convincingly (at least for the infection experiments in GBM cells), that it is important to include a time delay that represents the time from infection to cell death and production of new viral particles. Furthermore, the case is made that the time delay effect and sequestration of the virus in the infected cells leads to second order effects which further slow the spread of the virus.

Although the model is rather simplistic (it has radial symmetry, no vasculature, infection starting from a single point) and most kinetic rates are only known imprecisely, the agreement of the model prediction with the experimental data on the front speed of the VSV action is reassuring that the mathematical description of the augmented model is biologically plausible. The conclusions, of course, would have been stronger had the authors used an independent experimental model to validate their finding. Also, there is no notion of uncertainty in the predictions shown in Fig. 2. It would be useful if a sensitivity analysis could be included to demonstrate that model 3 is indeed the only model (among the 3 ) that is consistent with the experimental data.

Also, it is somewhat surprising that the authors did not also visualize the solutions of their models as radial density 'snapshots' at various endpoints. This (together with the parameter values used) could help others to reproduce their results.

Authors' response: We thank the review by Dr. G. Luebeck, which is quite positive with our research manuscript. We have reviewed the text according to his suggestions.
As suggested, we have improved Fig. 2 by adding lower and upper bounds to the model predictions obtained by considering the whole range of the parameter uncertainties. The new figure shows how important it is to include the second-order terms, because neither model 1 nor model 2 can explain the experimental data. The robustness of this conclusion has improved with this sensitivity analysis.

A new Fig. 3 has been added to the manuscript following the advice of both referees. It shows three snapshots of the populations of virus and infected cells in space at different instant. This new figure provides a visualization of the expansion process, as well as a new way to compute the front speed for model 3.

Minor points have been take into account and corrected in the text.

\section{Abbreviations}

GBM: Glioblastoma; VSV: vesicular stomatitis virus; PDE: partial differential equation; PFU: plaque-forming unit; BHK: baby hamster kidney; HPI: hours post infection; GFP: green fluorescent protein.

\section{Competing interests}

The authors declare that they have no competing interests.

\section{Authors' contributions}

VLR: participated in the design of the model, did the modeling, the analytic calculations and simulations and drafted the manuscript. NI: supervised the application of the analytical model and parameter selection, did the computational simulations and revised and edited the manuscript. JF: designed the mathematical model, supervised the implementation and participated in writing the manuscript. All authors read and approved the manuscript.

\section{Acknowledgments}

This work was partially funded by ICREA (Academia award) and the MINECO (projects SimulPast-CSD2010-00034, FIS-2009-13050 and FIS-2012-31307).

Received: 23 September 2015 Accepted: 11 December 2015

Published online: 07 January 2016

References

1. Kelly E, Russell SJ. History of oncolytic viruses: genesis to genetic engineering. Mol Ther. 2007;15(4):651-9.

2. Ledford H. Cancer-fighting viruses near market. Nature. 2015;526(7575): 622-23.

3. Novozhilov AS, Berezovskaya FS, Koonin EV, Karev GP. Mathematical modeling of tumor therapy with oncolytic viruses: Regimes with complete tumor elimination within the framework of deterministic models. Biol Direct. 2006;1:6. doi:10.1186/1745-6150-1-6.

4. Karev GP, Novozhilov AS, Koonin EV. Mathematical modeling of tumor therapy with oncolytic viruses: effects of parametric heterogeneity on cell dynamics. Biol Direct. 2006;1:30. doi:10.1186/1745-6150-1-30.

5. Wodarz D, Hofacre A, Lau JW, Sun Z, Fan H, Komarova NL. Complex spatial dynamics of oncolytic viruses in vitro: mathematical and experimental approaches. PLoS Comp Biol. 2012;8(6):e1002547. doi:10.1371/journal.pcbi.1002547.

6. Fort J, Solé RV. Accelerated tumor invasion under non-isotropic cell dispersal in glioblastomas. New J Phys. 2013;15:055001-10.

7. Özduman K, Wollmann G, Piepmeier JM, van den Pol AN. Systemic vesicular stomatitis virus selectively destroys multifocal glioma and metastatic carcinoma in brain. J Neurosci. 2008;28(8):1882-93. doi:10.1523/JNEUROSCI.4905-07.2008.

8. Wollmann G, Ozduman K, van den Pol AN. Oncolytic virus therapy for glioblastoma multiforme: concepts and candidates. Cancer J. 2012;18(1): 69-81. doi:10.1097/PPO.0b013e31824671c9. 
9. Wollmann G, Tattersall P, van den Pol AN. Targeting human glioblastoma cells: comparison of nine viruses with oncolytic potential. J Virol. 2005;79(10):6005-22.

10. Freeman Al, Zakay-Rones Z, Gomori JM, Linetsky E, Rasooly L, Greenbaum E, et al. Phase I/II trial of intravenous NDV-HUJ oncolytic virus in recurrent glioblastoma multiforme. Mol Ther. 2006;13(1):221-8.

11. Wollmann G, Rogulin V, Simon I, Rose JK, van den Pol AN. Some attenuated variants of vesicular stomatitis virus show enhanced oncolytic activity against human glioblastoma cells relative to normal brain cells. J Virol. 2010;84(3):1563-73. doi:10.1128/JVI.02040-09.

12. Russell SJ, Peng K-W, Bell JC. Oncolytic virotherapy. Nat Biotechnol. 2012;30(7):658-70. doi:10.1038/nbt.2287.

13. Brauer F, Castillo-Chavez C. Mathematical models in population biology and epidemiology. New York: Springer. 2001123-125.

14. Koch AL. The growth of viral plaques during the enlargement phase. J Theor Biol. 1964;6(03):413-431.

15. Yin J, McCaskill JS. Replication of viruses in a growing plaque: a reaction-diffusion model. Biophys J. 1992;61(6):1540-1549. doi:10.1016/S0006-3495(92)81958-6.

16. Haseltine EL, Lam V, Yin J, Rawlings JB. Image-guided modeling of virus growth and spread. Bull Math Biol. 2008;70(6):1730-48. doi:10.1007/s11538-008-9316-3.

17. Amor DR, Fort J. Virus infection speeds: Theory versus experiment. Phys Rev E. 2010;82:061905. doi:10.1103/PhysRevE.82.061905.

18. Wein LM, Wu JT, Kirn DH. Validation and analysis of a mathematical model of a replication-competent oncolytic virus for cancer treatment: implications for virus design and delivery. Cancer Res. 2003;63(6):1317-24.

19. Mok W, Stylianopoulos T, Boucher Y, Jain RK. Mathematical modeling of herpes simplex virus distribution in solid tumors: implications for cancer gene therapy. Clin Cancer Res. 2009;15(7):2352-60. doi:10.1158/1078-0432.CCR-08-2082.

20. Nowak M, May RM. Virus dynamics: Mathematical principles of Immunology and Virology. Oxford: Oxford University Press; 2000, pp. 100-109.

21. Beretta E, Kuang Y. Modeling and analysis of a marine bacteriophage infection. Math Bioscienc. 1998;149:57-76.

22. Wollmann G, Robek MD, van den Pol AN. Variable deficiencies in the interferon response enhance susceptibility to vesicular stomatitis virus oncolytic actions in glioblastoma cells but not in normal human glial cells. J Virol. 2007;81(3):1479-91.

23. de Rioja VL, Fort J, Isern N. Front propagation speeds of T7 virus mutants. J Theor Biol. 2015;385:112-118. doi:10.1016/j.jtbi.2015.08.005.

24. Gourley SA, Kuang Y. A delay reaction-diffusion model of the spread of bacteriophage infection. SIAM J Appl Math. 2005;65(2):550-566. doi:10.1137/S0036139903436613.

25. Jones DA, Smith HL, Thieme HR, Röst G. On spread of phage infection of bacteria in a petri dish. SIAM J Appl Math. 2012;72(2):670-688. doi:10.1137/110848360.

26. Fort J, Mendez V. Time-delayed spread of viruses in growing plaques. Phys Rev Lett. 2002;89(17):178101.

27. Isern N, Fort J. Time-delayed reaction-diffusion fronts. Phys Rev E. 2009;80:057103.

28. Ebert U, van Saarloos W. Front propagation into unstable states: universal algebraic convergence towards uniformly translating pulled fronts. Physica D. 2000;146:1-99.

29. Fisher RA. The wave of advance of advantageous genes. Ann Eugenics. 1937;7:353-369.

30. Stepien TL, Rutter EM, Kuang Y. A data-motivated density-dependent diffusion model of in vitro glioblastoma growth. Math Biosci Eng. 2015;12(6):1157-1172. doi:10.3934/mbe.2015.12.1157.

31. Ware BR, Raj T, Flygare WH, Lesnaw JA, Reichmann ME. Molecular Weights of Vesicular Stomatitis Virus and Its Defective Particles by Laser Light-Scattering Spectroscopy. J Virol. 1973;11(1):141-145.

32. Stein AM, Vader DA, Deisboeck TS, Chiocca EA, Sander LM, Weitz DA. Directionality of glioblastoma invasion in a 3D in vitro experiment. arXiv, http://arxiv.org/pdf/q-bio/0610031.pdf. Accessed 30 Jul 2015.

33. Stein AM, Demuth $T$, Mobley D, Berens M, Sander LM. A mathematical model of glioblastoma tumor spheroid invasion in a three-dimensional in vitro experiment. Biophys J. 2007;92(1):356-65.

34. Rockne R, Rockhill JK, Mrugala M, Spence AM, Kalet I, Hendrickson K, et al. Predicting the efficacy of radiotherapy in individual glioblastoma patients in vivo: a mathematical modeling approach. Phys Med Biol. 2010;55(12):3271-85. doi:10.1088/0031-9155/55/12/001.

35. Friedman A, Tian JP, Fulci G, Chiocca EA, Wang J. Glioma virotherapy: effects of innate immune suppression and increased viral replication capacity. Cancer Research. 2006;66(4):2314-19.

36. Eikenberry SE, Sankar T, Preul MC, Kostelich EJ, Thalhauser CJ, Kuang T. Virtual glioblastoma: growth, migration and treatment in a three-dimensional mathematical model. Cell Prolif. 2009;42(04):511-528. doi:10.1111/j.1365-2184.2009.00613.x.

37. van den Pol AN, Davis JN. Highly attenuated recombinant vesicular stomatitis virus VSV-12'GFP displays immunogenic and oncolytic activity. J Virol. 2013;87(2):1019-34. doi:10.1128/JVI.01106-12.

38. Shishido K, Watarai A, Naito S, Ando T. Action of bleomycin on the bacteriophate T7 infection. J Antibiot (Tokyo). 1975;28(9):676-80.

39. Koks CAE, De Vleeschouwer S, Graf N, Van Gool SW. Immune Suppression during Oncolytic Virotherapy for High-Grade Glioma; Yes or No? J Cancer. 2015;6(3):203-217. doi:10.7150/jca.10640.

40. Russell SJ, Peng K-W, Bell JC. Oncolytic virotherapy. Nature Biotech. 2012;30(7):658-70. doi:10.1038/nbt.2287.

41. Mahoney DJ, Stojdl DF, Laird G. Virus therapy for cancer. Sci Am. 2014;311(5):54-9.

\section{Submit your next manuscript to BioMed Central and we will help you at every step:}

- We accept pre-submission inquiries

- Our selector tool helps you to find the most relevant journal

- We provide round the clock customer support

- Convenient online submission

- Thorough peer review

- Inclusion in PubMed and all major indexing services

- Maximum visibility for your research

Submit your manuscript at www.biomedcentral.com/submit

C) Biomed Central 